\title{
CISS Effect: A Magnetoresistance Through Inelastic Scattering
}

\author{
Karssien Hero Huisman* and Joseph Marie Thijssen
}

Cite This: J. Phys. Chem. C 2021, 125, 23364-23369

Read Online

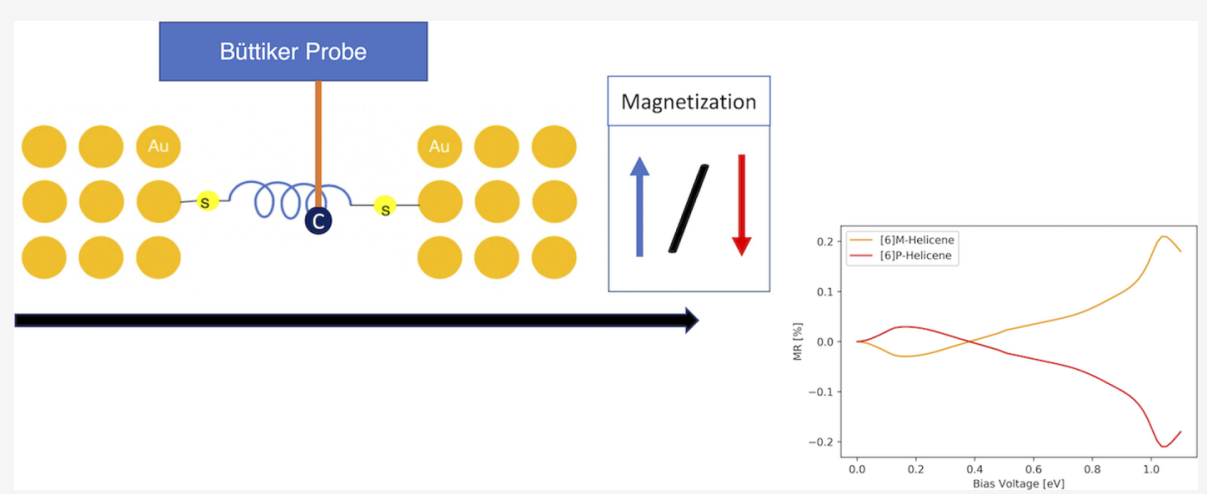

ABSTRACT: One of the manifestations of chirality-induced spin selectivity is the magnetoresistance (MR) in two-terminal transport measurements on molecular junctions. This paper investigates the effect of spin-orbit coupling in the leads on the polarization of the transmission. A helicene molecule between two gold contacts is studied using a tight binding model. To study the occurrence of $\mathrm{MR}$, which is prohibited in coherent transport, as a consequence of the Büttiker reciprocity, we add Büttiker probes to the system in order to incorporate inelastic scattering effects. We show that for a strict two-terminal system without inelastic scattering, the MR is strictly zero in the linear and nonlinear regimes. We show that for a two-terminal system with inelastic scattering, a nonzero MR does appear in the nonlinear regime, reaching values of the order of $0.1 \%$. Our calculations show that for a two-terminal system respecting time-reversal symmetry and charge conservation, a nonzero MR can only be obtained through inelastic scattering. However, spin-orbit coupling in the leads in combination with inelastic scattering modeled with the Büttiker probe method cannot explain the magnitude of the MR measured in experiments.

\section{INTRODUCTION}

In two-terminal transport experiments, ${ }^{1-9}$ polarizations of the current, that is, magnetoresistances (MRs, see eq 2 for the definition) ranging from 6 to $90 \%$ have been measured for selfassembled monolayers (SAMs) of chiral molecules. This MR is a manifestation of the connection between chirality of the molecular structure and spin selectivity, commonly denoted as the CISS effect. Theoretical efforts have been geared toward explaining the existence of CISS in transport including quantitative estimates for the MR.

Most theoretical work conducted on CISS focuses on calculating the spin-polarization of the transmission (SPT, see eq 1). The spin-orbit coupling of the molecule's constituents is too weak to explain the CISS effect. In ref 10, the molecule helicene was studied and a maximum SPT of the order $10^{-5} \%$ was found. When decoherence is taken into account, by adding onsite imaginary terms to the Hamiltonian, a maximum SPT of $0.3 \%$ was found. As in this approach, the particle number is not conserved, the system is no longer strictly two-terminal and it therefore does not explain the MR unless in the experiment, electrons are leaking from the device into another reservoir than the source or drain. In two-terminal transport experi- ments, heavy metals such as gold, platinum, and silver are often used. These metals are known to have a strong spin-orbit coupling. Previous theoretical work has studied the effect of large spin-orbit coupling in gold leads on the $\mathrm{SPT}^{11,12}$ in density functional theory and tight binding models. ${ }^{13,14}$ SPTs reaching values of $1^{11}$ and $5 \%^{12}$ were found. Another interesting proposal for obtaining sizeable SPTs was put forward by Dalum and Hedegard. ${ }^{15}$ They noticed that close to degeneracies in the spectrum, the eigenstates of the Hamiltonian rotate away significantly from the unperturbed states, leading to substantial SPTs. Until recently, most theoretical work that tried to explain the CISS effect focused on calculating the SPT but it has now been realized that SPT does not necessarily lead to the occurrence of a MR.

Received: July 12, 2021

Revised: $\quad$ September 21, 2021

Published: October 18, 2021 
Two fundamental reciprocity theorems are very important when considering the nonzero MR measured in experiments and putting it in a theoretical perspective. The first is the Onsager-Casimir reciprocity ${ }^{16,17}$ which implies that for a twoterminal interacting system the MR can only be nonzero in the nonlinear regime. The second is the Büttiker reciprocity theorem. ${ }^{18}$ This theorem that is based on time-reversal symmetry and unitarity of the S-matrix prohibits $M R$ to occur for two-terminal non-interacting systems irrespective of the regime (linear/nonlinear, see Section 2.2), even when the SPT is nonzero. In order to obtain a nonzero MR, interactions must be included. In refs 19 and 20, the effects of the Coulomb interactions and a coupling to vibrational modes were studied, respectively, and a nonzero MR was found.

Nonzero MR can be obtained by breaking charge conservation but in the experiment the leakage currents are often too small to justify this as a cause of a MR. In this paper, we focus on another option: electrons that scatter inelastically while conserving their number during the process. We construct a tight binding model for a junction in which helicene is coupled to two gold electrodes which have a strong spin-orbit coupling. Büttiker probes are added in order to model inelastic scattering while maintaining charge conservation. We denote the system with Büttiker probes as an interacting system and the system without Büttiker probes as a non-interacting system. Tight binding has the advantage over a quantum-chemical calculation that inelastic effects can easily be included through the Büttiker probes. Also, it is easy to study the effect of changing couplings and interaction parameters.

We first validate our model by calculating the SPT and comparing this to density functional theory results. ${ }^{11,12}$ The first goal of this paper is to verify the occurrence of a MR and to investigate its magnitude when the Büttiker probes are active. The second goal is to address the characteristic features of the $\mathrm{MR}$ and compare it with the characteristics found in the experiment.

In Section 2.1, the SPT is defined. In Section 2.2, the MR is defined and it is explained what restrictions are imposed on the MR by the Büttiker and Onsager-Casimir reciprocity together with the implications for experimental observables. In Section 2.3, a description of the non-interacting system is given. In Section 2.4, the Büttiker probes and their parameters are described. The results for the non-interacting and interacting system are given in Sections 3.1 and 3.2, respectively. In Section 4, we present conclusions.

\section{COMPUTATIONAL DETAILS}

2.1. Theory: SPT. The SPT from the right to the left lead is defined as

$$
P_{z}(E)=\frac{T_{\mathrm{RL}}^{\uparrow \uparrow}+T_{\mathrm{RL}}^{\downarrow \uparrow}-T_{\mathrm{RL}}^{\uparrow \downarrow}-T_{\mathrm{RL}}^{\downarrow \downarrow}}{T_{\mathrm{RL}}}
$$

Here, $T_{\mathrm{RL}}^{\mathrm{ss}}$ is the energy-dependent transmission probability for an electron in the right lead with spin $\mathrm{s}=\uparrow, \downarrow$ (quantised in the $z$-direction, which we take as the direction of the transmission) to be transmitted to the left lead with spin $\mathrm{s}^{\prime}=\uparrow, \downarrow$ and $T_{\mathrm{RL}}=$ $\sum_{\mathrm{s}, \mathrm{s}^{\prime} \in\{\uparrow, \downarrow\}} T_{\mathrm{RL}}^{\mathrm{ss}}$, with $T_{\mathrm{RL}}$ the total transmission probability from right to left. In the non-equilibrium Green's function formalism, the transmission function is given by the MeirWingreen formula $T_{\mathrm{RL}}(E)=\operatorname{Tr}\left[\boldsymbol{G}(\boldsymbol{E}) \boldsymbol{\Gamma}_{\mathbf{R}} \mathbf{G}(\boldsymbol{E})^{\dagger} \boldsymbol{\Gamma}_{\mathbf{L}}(\boldsymbol{E})\right]$, with $\boldsymbol{\Gamma}_{\mathrm{L}, \mathrm{R}}(E)$ the imaginary part of the self-energy of the left and right lead, respectively, and $\mathbf{G}(E)$ the retarded Green's function which is given by $\mathbf{G}(E)=\left(E \mathbf{1}-\mathbf{H}-\boldsymbol{\Lambda}_{\mathrm{L}}-\boldsymbol{\Lambda}_{\mathrm{R}}+\right.$ $\left.i / 2 \boldsymbol{\Gamma}_{\mathrm{L}}+i / 2 \boldsymbol{\Gamma}_{\mathrm{R}}\right)^{-1}$. Here, $\mathbf{1}$ is the unit-matrix, $\mathbf{H}$ is the Hamiltonian of the scattering region in Figure 1, and $\boldsymbol{\Lambda}_{\mathrm{L}, \mathrm{R}}$ the real part of the self energy of the left, right lead, respectively. Below, we drop the explicit dependence of the transmission on energy $E$.

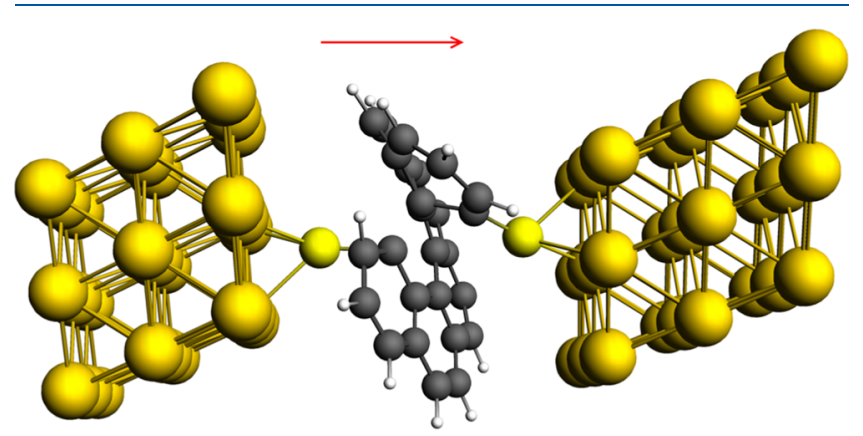

Figure 1. Reprinted with permission from ref 12. Copyright 2018, Rebergen. Schematic picture of the scattering region. The gold spheres are gold atoms, the bright yellow spheres are sulfur atoms, and the gray spheres in the middle are carbon atoms. To this scattering region, semi-infinite gold leads are attached.

2.2. MR and the Reciprocity Theorems. We define the MR as the normalized difference of the currents for the up and down magnetization $m,-m$ of the lead

$$
\mathrm{MR}=\frac{I(m)-I(-m)}{I(m)+I(-m)}
$$

For a two-terminal system which respects current conservation, the net current into the left lead is calculated with

$$
I(m, V)=\frac{e}{h} \int_{-\infty}^{\infty} T_{\mathrm{RL}}(m)\left(f_{\mathrm{L}}(E)-f_{\mathrm{R}}(E)\right) \mathrm{d} E
$$

where $f_{\mathrm{L}}(E), f_{\mathrm{R}}(E)$ are the Fermi-Dirac distributions of the left and right lead, respectively, and $m$ is the magnetization of the left lead. The chemical potentials for spin up and down electrons in the left/right lead are equal, that is, $\mu_{i}^{\uparrow}=\mu_{i}^{\downarrow}$ for $i=$ L, R. Assuming symmetric capacitive coupling to the left and right lead, the chemical potentials of the left and right leads are $E_{\mathrm{F}}+V / 2$ and $E_{\mathrm{F}}-V / 2$, respectively, with $E_{\mathrm{F}}$ the Fermi energy and $V$ the bias voltage. The current can be expanded in the bias voltage

$$
I(m, V)=G_{1}(m) V+G_{2}(m) V^{2}+G_{3}(m) V^{3}+\ldots
$$

We define $\Delta I(m, V)$ as the difference between the currents for positive and negative magnetization. Using eq 4 , we can write $\Delta I(m, V)$ as

$$
\begin{aligned}
\Delta I(m, V) \equiv & I(m, V)-I(-m, V) \\
= & {\left[G_{1}(m)-G_{1}(-m)\right] V+\left[G_{2}(m)-G_{2}(-m)\right] } \\
& V^{2}+\left[G_{3}(m)-G_{3}(-m)\right] V^{3}+\ldots
\end{aligned}
$$

We define the odd and even part of $\Delta I(m, V)$ as

$$
\begin{aligned}
A(m, V)= & \frac{1}{2}[\Delta I(m, V)-\Delta I(m,-V)] \\
= & {\left[G_{1}(m)-G_{1}(-m)\right] V+\left[G_{3}(m)-G_{3}(-m)\right] } \\
& V^{3}+\ldots
\end{aligned}
$$




$$
\begin{aligned}
B(m, V)= & \frac{1}{2}[\Delta I(m, V)+\Delta I(m,-V)] \\
= & {\left[G_{2}(m)-G_{2}(-m)\right] V^{2}+\left[G_{4}(m)-G_{4}(-m)\right] } \\
& V^{4}+\ldots
\end{aligned}
$$

respectively.

In ref 18 , it was shown that for a two-terminal system, timereversal symmetry and unitarity of the S-matrix (which expresses charge conservation) imply that $T_{\mathrm{RL}}(m)=$ $T_{\mathrm{RL}}(-m)$. This relation is known as the Büttiker reciprocity theorem for two-terminal systems. Combining this relation with eq 3, it trivially follows that: $I(m, V)=I(-m, V)$. Combining this with eq 5 , we obtain

$$
\begin{aligned}
\Delta I(m, V)= & {\left[G_{1}(m)-G_{1}(-m)\right] V+\left[G_{2}(m)-G_{2}(-m)\right] } \\
& V^{2}+\left[G_{3}(m)-G_{3}(-m)\right] V^{3}+\ldots \\
= & 0
\end{aligned}
$$

From this, it follows that: $G_{n}(m)=G_{n}(-m)$ for $n=1,2, \ldots$ if Büttiker reciprocity holds. Time-reversal symmetry and unitarity of the S-matrix prohibit a finite $\Delta I(m, V)$ in the linear and nonlinear regime for a two-terminal system without interactions.

On the other hand, the Onsager-Casimir reciprocity holds for systems with interactions. The Onsager-Casimir reciprocity implies that $I(m, V)=I(-m, V)$ in the linear regime: $G_{1}(m)=G_{1}(-m),{ }^{16,17}$ that is, the linear coefficient is an even function of the magnetization. This means that the differential conductance evaluated at $V=0$ is identical for opposite magnetizations

$$
G_{1}(m) \equiv \frac{\mathrm{d} I(m, V=0)}{\mathrm{d} V}=\frac{\mathrm{d} I(-m, V=0)}{\mathrm{d} V}=G_{1}(-m)
$$

For the non linear-coefficients: $G_{2}(m), G_{3}(m), \ldots$ in principle we can have: $G_{n}(m) \neq G_{n}(-m)^{21-23}$ for $n \geq 2$. In ref 23 , it was shown that electron interactions allow for a second order contribution (odd in $m$ ) $\alpha m V^{2}$ to the current from which clearly $G_{2}(m) \neq G_{2}(-m)$. Another example of this is the electrical magnetochiraleffect ${ }^{24}$ which gives (for small bias voltages) a second order contribution to the current ${ }^{14} G_{2}(m)=$ $G^{0}(\chi) m$ (with $G^{0}(\chi)$ a coefficient which depends on the chirality $\chi)$ for which also $G_{2}(m) \neq G_{2}(-m)$. In ref 25 , a rigorous analysis based on full counting statistics derived relations between the coefficients $G_{n}(m), G_{n}(-m)$ under microscopic reversibility. As expected, the Onsager-Casimir relation $G_{1}(m)=G_{1}(-m)$ was found. From this work, it can also be concluded that microscopic reversibility does not imply that the nonlinear coefficients for opposite magnetizations are equal: $G_{n}(m) \neq G_{n}(-m)$ for $n \geq 2$.

We now expand the MR (eq 2) in the bias voltage for an interacting system. Defining $\Delta G_{n}(m) \equiv G_{n}(m)-G_{n}(-m)$ and $\bar{G}_{n}(m) \equiv G_{n}(m)+G_{n}(-m)$, we can write the MR as

$$
\operatorname{MR}=\frac{\Delta G_{1}(m) V+\Delta G_{2}(m) V^{2}+\ldots}{\bar{G}_{1}(m) V+\bar{G}_{2}(m) V^{2}+\ldots}
$$

The Onsager-Casimir reciprocity implies $\Delta G_{1}(m)=0$, so that eq 10 simplifies to: $\mathrm{MR}=\frac{\Delta G_{2}(m) V+\ldots}{\bar{G}_{1}(m)+\bar{G}_{2}(m) V+\ldots}$, showing that the MR vanishes at zero bias. Remarkably in ref 1 , a nonzero MR was found at zero bias which seems in contradiction with the Onsager-Casimir reciprocity.
The analysis in this section and refs $21-23$ and 25 imply that interactions play a crucial role in obtaining a MR.

2.3. Tight Binding Hamiltonian. Our Slater-Koster tight binding model addresses four parts of a transport junction (i) semi-infinite gold leads without spin-orbit coupling, (ii) a block of $3 \times 3 \times 3$ gold atoms with spin-orbit coupling, (iii) a sulfur anchoring group, and (iv) a helicene molecule. A schematic picture of the scattering region is shown in Figure 1. The outer $3 \times 3$ layers of gold atoms on the left and right side each have a semi-infinite gold lead without spin-orbit coupling attached to it see Figure 1 .

For parts (i,ii), the Slater-Koster tight binding parametrization of ref 26 will be used; for a detailed description, see Section 1.1 of the Supporting Information. The tight binding parameters reported in ref 27 are used for the anchoring group. For a detailed description of part (iii), see Supporting Information Section 1.3. We use the full SlaterKoster tight binding model of helicene reported in ref 10 and the Slater-Koster tight binding parameters are given in the Supporting Information Section 1.2. Only the $2 \mathrm{p}, 2 \mathrm{~s}$ orbitals of carbon atoms are considered. A $[n]$ helicene molecule consist of a chain of $n$ benzene rings. Helicene has two enantiomeric states: $\mathrm{P}$ and $\mathrm{M}$ related by mirror symmetry. The numerical calculations were performed using the Kwant code. ${ }^{28}$ For details regarding the implementation in Kwant, see Section 1.5 of the Supporting Information. Our Python code is available on https://github.com/khhuisman/CISS_effect.

2.4. Büttiker Probes. In order to model interactions, we use the Büttiker probes; virtual leads that are attached to each site of the molecule. The Büttiker probes can act as a "dephasing probe" or a "voltage probe" to model elastic and inelastic processes, respectively. In ref 29, it was shown that for an Aharonov-Bohm junction, a dephasing probe does not lead to a finite $\mathrm{MR}$ in the linear, nor in the nonlinear regime, but a voltage probe does lead to a nonzero $\mathrm{MR}$ in the nonlinear regime, confirming the findings of ref 22. Therefore, in our model, we use voltage probes. The Büttiker probe acts as a voltage probe if the total charge current into the probe is zero. The chemical potential of the probe is determined in a selfconsistent manner such that the total charge current into the probe is zero. However, there is a finite net energy current going into each virtual lead which represents energy being dissipated into an external environment (the Büttiker probe), while respecting charge conservation (in contrast to refs 10 and 14 where the addition of onsite imaginary terms leads to loss of particles). The Hamiltonian of a virtual lead is modeled as a semi-infinite linear chain (see Supporting Information Section 1.4).

We define a probe to be a collection of virtual leads. For the [6] helicene molecule, we have 26 carbon atoms to which we can attach a virtual lead. One probe containing several leads, with one and the same chemical potential, can be attached to several sites. Alternatively, a virtual lead, characterized by a single chemical potential, can be connected to more than one carbon atoms.

The hopping parameter within the lead is $t_{0}$ and $t_{\text {leak }}$ is the coupling between the lead and the carbon atom. In order to cover a wide range of energies, we choose $t_{0}$ to be large compared to the Slater-Koster tight binding hopping parameters of carbon (see Supporting Information Section 1.2), hence, we take $t_{0}=50 \mathrm{eV}$. We distinguish three regimes: $t_{\text {leak }}>t_{0}, t_{\text {leak }}=t_{0}$, and $t_{\text {leak }}<t_{0}$. 


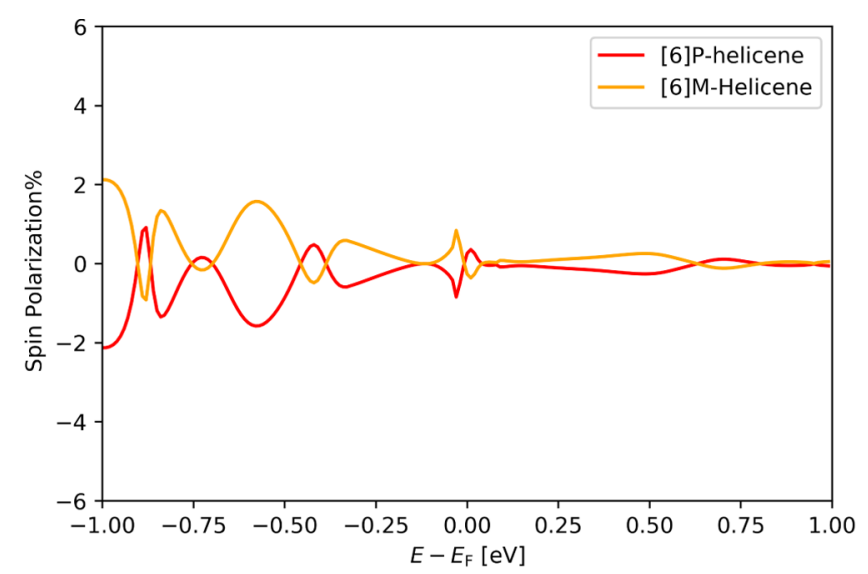

(a)

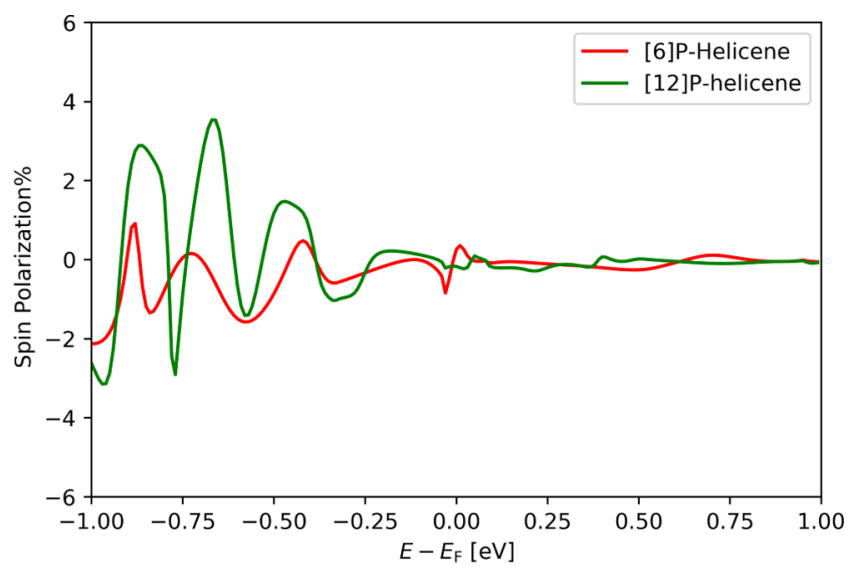

(b)

Figure 2. (a) Spin-polarization for the gold-sulfur-[6]P and $\mathrm{M}$ helicene unmagnetized junction as a function of energy in red, orange respectively. $E_{\mathrm{F}}$ is the Fermi energy of gold in electronvolt $(\mathrm{eV})$. (b) Spin-polarization for $[6,12]$ P-helicene in the unmagnetized junction as a function of energy. $E_{\mathrm{F}}$ is the Fermi energy of gold in $\mathrm{eV}$. The figure shows an increase in spin-polarization when the length of the molecule is increased.

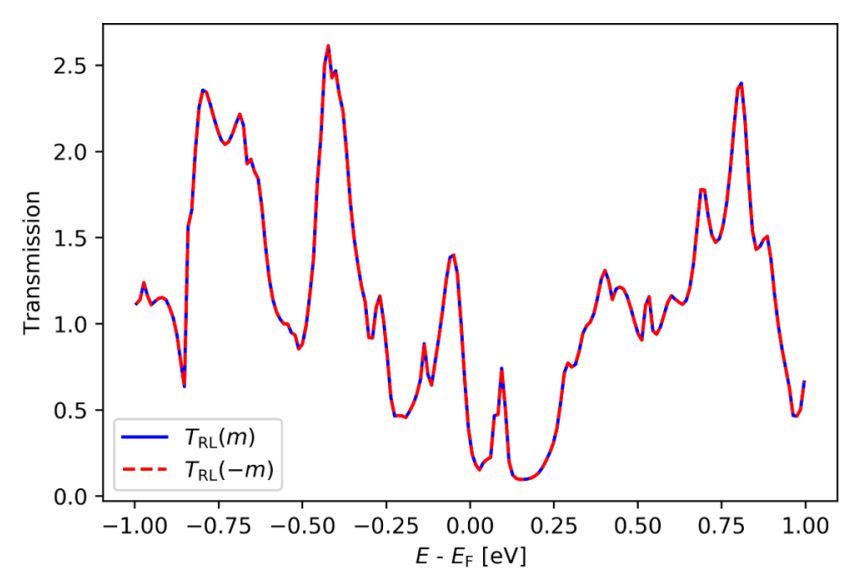

(a)

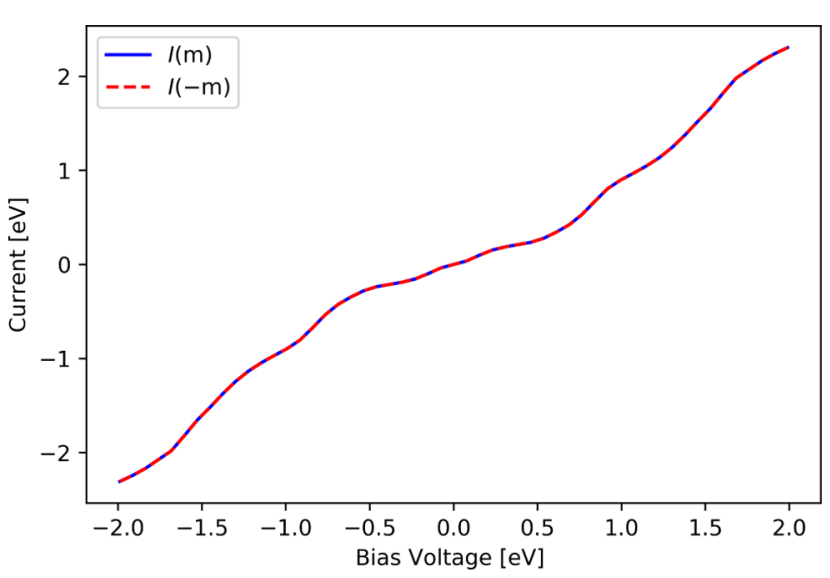

(b)

Figure 3. (a) Transmission from right to left for $E_{\mathrm{F}}=-5.3 \mathrm{eV}$, and the magnetizations $m= \pm 2.1$ of the left lead. The difference between $T_{\mathrm{RL}}(m)$ and $T_{\mathrm{RL}}(-m)$ is zero. (b) Charge current $I(m)$ in $\mathrm{eV}$ into the left lead for the magnetizations $\pm m$ increases and shows no difference between $I(m)$, $I(-m)$.

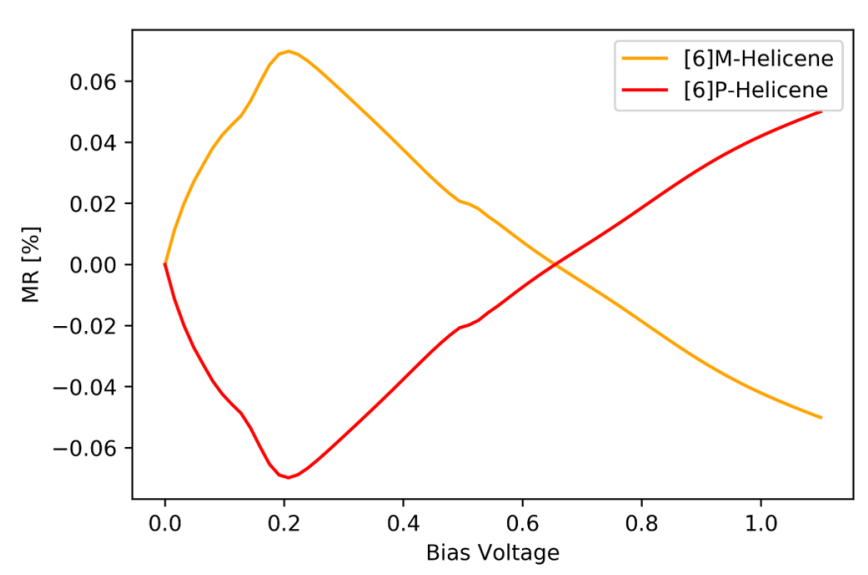

(a)

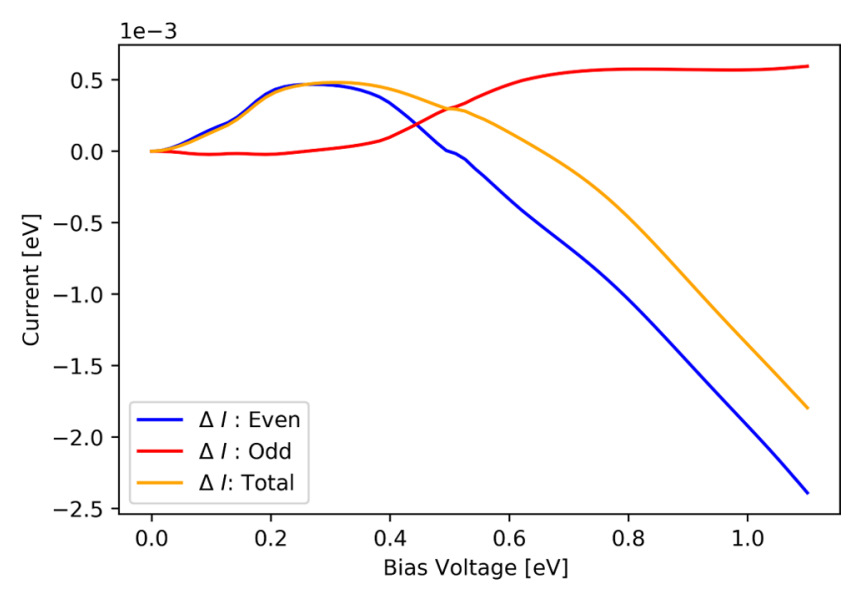

(b)

Figure 4. (a) MR in percentage for the gold-sulfur-[6] $\mathrm{P}$ and $\mathrm{M}$ helicene junction with 1 probe consisting of 1 lead attached, as a function of bias voltage in red and orange respectively. The MR changes sign between the enantiomers of the molecule. (b) Even and odd part in $V$ of $\Delta I(m, V)$ in blue and red, respectively, as a function of bias voltage. 
We varied the attachment position of the probe(s), the amount of leads in a single probe, the coupling between the probe and the molecule: $t_{\text {leak }}$ and the amount of independent probes consisting of one lead. In Supporting Information Section 3, we prove that a voltage probe always satisfies the Onsager-Casimir reciprocity: $G_{1}(m)=G_{1}(-m)$.

\section{RESULTS}

3.1. Two-Terminal Junction, Non-interacting. In Figure 2a, the SPT (see eq 1) for [6]P,M-helicene is shown for an unmagnetized lead and we find a maximum absolute value of the SPT of $2 \%$, which is comparable with the density functional theory studies ${ }^{11,12}$ (see Supporting Information Section 1.6 for the result of the ref 12). In Figure 2a, we also see that the SPT is exactly opposite in sign for [6]P,M-helicene as expected (see Supporting Information Section 2). In Figure $2 \mathrm{~b}$, the SPT for [12]P-helicene and [6]P-helicene for an unmagnetized lead is plotted. The SPT is seen to increase with the length of the molecule as was found in ref 11 . Dalum and Hedegard $^{15}$ find an increased SPT at degeneracies of the molecular spectrum. The frontier levels do not show degeneracies beyond the usual Kramer's degeneracy. Moreover, the spin-orbit coupling of the gold supersedes the effect highlighted by these authors.

We now turn to a system with a magnetized lead. In Figure $3 \mathrm{a}$, the transmissions $T_{\mathrm{RL}}(m), T_{\mathrm{RL}}(-m)$ are plotted in blue and red, respectively, as a function of energy. We see that for every energy, these transmissions are equal: $T_{\mathrm{RL}}(m)=T_{\mathrm{RL}}(-m)$, confirming that the Büttiker reciprocity holds for our twoterminal system. The currents $I(m), I(-m)$ are plotted in Figure $3 \mathrm{~b}$ in blue and in red, respectively, as a function of bias voltage $V$ and are calculated using eq 3 for a Fermi energy of gold $E_{\mathrm{F}}=-5.3 \mathrm{eV}$ at $T=0 \mathrm{~K}$. They are obviously identical, confirming that the MR for this non-interacting system is zero in the linear and nonlinear regime, even though there is a nonzero SPT in the bias window $\left[E_{\mathrm{F}}-V / 2, E_{\mathrm{F}}+V / 2\right]$ for the magnetized system (not plotted).

3.2. Two-Terminal Junction, Inelastic Scattering. Varying the attachment position, the order of magnitude of the MR varies between of $10^{-2}$ and $10^{-1} \%$. In Figure $4 \mathrm{a}$, the $\mathrm{MR}$ is plotted for [6]M,P-helicene. We see that its sign changes between the enantiomers of the molecule, as was found in experiments. ${ }^{2,4,8,9}$ In Figure 4a, there is a MR around zero bias (except at $V=0$ there $M R=0$ as expected see Section 2.2), which is allowed by the Onsager-Casimir because the MR only arises in the nonzero difference between higher order contributions $\left(\Delta G_{n}\right.$ for $\left.n \geq 2\right)$ to $\Delta I(m, V)$ see eq 10 .

In Figure $4 \mathrm{~b}$, we show the even and odd parts of $\Delta I(m, V)$ in blue and in red, see eqs 6 and 7. The fact that the voltage probe satisfies the Onsager-Casimir reciprocity (see Supporting Information 3 ) ensures that there are no contributions to $\Delta I(m, V)$ which are linear in $V$. This means that the odd part in Figure $4 \mathrm{~b}$ must be nonlinear in nature. The even part of the $\Delta I(m, V)$ in Figure $4 \mathrm{~b}$ can only scale with $V^{2}, V^{4}, \ldots$ which by definition is nonlinear. In Figure $4 b$, we see the even contribution to $\Delta I(m, V)$ dominates. Experimental results on CISS imply that $\Delta I(m, V)$ has an odd part which is larger than the even contribution. ${ }^{1,4-9}$

One might expect that adding more independent probes leads to more inelastic scattering and therefore a larger $M R$, however, this turns out not to be the case. We calculated the MR for up to and including four independent probes and found that the MR did not increase with the number of independent probes. The order of magnitude of the largest MR stayed below $0.1 \%$. We also varied the amount of leads in one independent probe. For this case, the amount of probes did not change the order of magnitude of the MR.

We found that the MR primarily depends on the spin-orbit coupling of the gold whereas the effect of the spin-orbit coupling from the carbon atoms on the MR is negligible, and it vanishes when the spin-orbit coupling is switched off altogether. So far, the potential across the molecule has been kept constant. If we apply a linear drop to the onsite potential along the helix axis of the molecule, the maximum MR remains of the same order of $0.1 \%$.

Fransson ${ }^{19}$ has studied a model capturing the coupling between the electrons and the vibrational modes. He obtained a significant MR only when including a coupling to the spinorbit hopping term in the Hamiltonian. This term has a rather specific form and our generic Büttiker probes may fail to represent this. Fransson found that the MR scales with the coupling between electrons and vibrational modes. In contrast, the Büttiker probe method used here gives a MR which quickly saturates to a (rather low) MR value when increasing the coupling parameter $t_{\text {leak }}$. Further research into the specific form of the self-energy and the construction of an appropriate Büttiker probe seems useful. The question remains why substantial $\mathrm{MR}$ is found in experiments. Maybe this is a consequence of interactions such as the ones we study in this paper, but it can also be that in the experiments on SAMs, part of the current is leaking into reservoirs other than the two terminals of the voltage source.

It could be that SAMs of chiral molecules allow for molecule-molecule or molecule-substrate interactions. These interactions might manifest themselves as a magnetization of the substrate as was measured in ref 30 . How this magnetization effects the currents in two-terminal junctions is unclear. Whether CISS is a single molecule property or rather a property of SAMs remains an open question. More experiments on single-molecule (rather than SAMs), twoterminal junctions are highly desirable.

\section{CONCLUSIONS}

We have constructed a tight binding model that includes spinorbit coupling in the gold leads and found a SPT comparable to that found in density functional theory studies on a similar system. ${ }^{11,12}$ Furthermore the SPT changes sign between the enantiomers of the molecule and increases with the length of the molecule. In line with the Büttiker reciprocity theorem, we find that the MR vanishes in the linear and nonlinear regime in the non-interacting system even though the spin polarized transmission is finite.

Adding Büttiker probes yields a MR beyond the linear regime. The spin-orbit coupling in the leads in combination with inelastic scattering modeled through local Büttiker probes gives a nonzero $\mathrm{MR}$ of the order $0.1 \%$, which is $1-2$ orders of magnitude smaller than the MR found in the experiments. ${ }^{1-9}$ The MR only arises in the nonzero difference between higher order contributions $\left(\Delta G_{n} \neq 0\right.$ for $\left.n \geq 2\right)$, satisfying the Onsager-Casimir reciprocity. Furthermore, we find that the MR changes sign between the enantiomers of the molecule as was found in refs 248 , and 9 and contrary to experiment $\Delta I(m, V)$ has an even contribution in bias voltage which is larger than the odd contribution. 


\section{ASSOCIATED CONTENT}

\section{(s) Supporting Information}

The Supporting Information is available free of charge at https://pubs.acs.org/doi/10.1021/acs.jpcc.1c06193.

Tight binding Hamiltonian, sign change of SPT. Büttiker probes and Onsager reciprocity, and charge current formula (PDF)

\section{AUTHOR INFORMATION}

\section{Corresponding Author}

Karssien Hero Huisman - Kavli Institute of Nanoscience,

Delft University of Technology, 2628 CJ Delft, The

Netherlands; 이이.org/0000-0002-3358-9400;

Phone: +31651964048; Email: k.h.huisman@tudelft.nl

\section{Author}

Joseph Marie Thijssen - Kavli Institute of Nanoscience, Delft University of Technology, 2628 CJ Delft, The Netherlands

Complete contact information is available at:

https://pubs.acs.org/10.1021/acs.jpcc.1c06193

\section{Notes}

The authors declare no competing financial interest.

\section{ACKNOWLEDGMENTS}

This publication is part of the project "CISS" (with project number 680.92.18.01) of the research programme "Natuurkunde Vrije Programma's" which is financed by the Dutch Research Council (NWO). We acknowledge useful discussions with $\mathrm{Xu}$ Yang and Bart van Wees.

\section{REFERENCES}

(1) Kulkarni, C.; Mondal, A. K.; Das, T. K.; Grinbom, G.; Tassinari, F.; Mabesoone, M. F. J.; Meijer, E. W.; Naaman, R. Highly Efficient and Tunable Filtering of Electrons' Spin by Supramolecular Chirality of Nanofiber-Based Materials. Adv. Mater. 2020, 32, 1904965.

(2) Aragonès, A. C.; Medina, E.; Ferrer-Huerta, M.; Gimeno, N.; Teixidó, M.; Palma, J. L.; Tao, N.; Ugalde, J. M.; Giralt, E.; DíezPérez, I.; et al. Measuring the Spin-Polarization Power of a Single Chiral Molecule. Small 2017, 13, 1602519.

(3) Kiran, V.; Cohen, S. R.; Naaman, R. Structure Dependent Spin Selectivity in Electron Transport Through Oligopeptides. J. Chem. Phys. 2017, 146, 092302.

(4) Kiran, V.; Mathew, S. P.; Cohen, S. R.; Hernández Delgado, I.; Lacour, J.; Naaman, R. Helicenes-A New Class of Organic Spin Filter. Adv. Mater. 2016, 28, 1957.

(5) Xie, Z.; Markus, T. Z.; Cohen, S. R.; Vager, Z.; Gutierrez, R.; Naaman, R. Correction to Spin Specific Electron Conduction through DNA Oligomers. Nano Lett. 2012, 12, 523.

(6) Al-Bustami, H.; Koplovitz, G.; Primc, D.; Yochelis, S.; Capua, E.; Porath, D.; Naaman, R.; Paltiel, Y. Single Nanoparticle Magnetic Spin Memristor. Small 2018, 14, 1801249.

(7) Bullard, G.; Tassinari, F.; Ko, C.-H.; Mondal, A. K.; Wang, R.; Mishra, S.; Naaman, R.; Therien, M. J. Low-Resistance Molecular Wires Propagate Spin-Polarized Currents. J. Am. Chem. Soc. 2019, 141, 14707-14711.

(8) Tassinari, F.; Jayarathna, D. R.; Kantor-Uriel, N.; Davis, K. L.; Varade, V.; Achim, C.; Naaman, R. Chirality Dependent Charge Transfer Rate in Oligopeptides. Adv. Mater. 2018, 30, 1706423.

(9) Bloom, B. P.; Kiran, V.; Varade, V.; Naaman, R.; Waldeck, D. H. Spin Selective Charge Transport Through Cysteine Capped Cdse Quantum Dots. Nano Lett. 2016, 16, 4583-4589.

(10) Geyer, M.; Gutierrez, R.; Mujica, V.; Cuniberti, G. ChiralityInduced Spin Selectivity in a Coarse-Grained Tight-Binding Model for Helicene. J. Phys. Chem. C 2019, 123, 27230-27241.
(11) Zöllner, M. S.; Varela, S.; Medina, E.; Mujica, V.; Herrmann, C. Insight Into the Origin of Chiral-Induced Spin Selectivity From a Symmetry Analysis of Electronic Transmission. J. Chem. Theory Comput. 2020, 16, 2914-2929.

(12) Rebergen, M. A. Study of Induced Spin Selectivity in Chiral Molecules, MA Thesis, Delft University of Technology, 2018.

(13) Gersten, J.; Kaasbjerg, K.; Nitzan, A. Induced Spin Filtering in Electron Transmission Through Chiral Molecular Layers Adsorbed on Metals With Strong Spin-Orbit Coupling. J. Chem. Phys. 2013, 139, 114111.

(14) Liu, Y.; Xiao, J.; Koo, J.; Yan, B. Chirality-Driven Topological Electronic Structure of DNA-Like Materials. Nat. Mater. 2021, 20 , 638

(15) Dalum, S.; Hedegård, P. Theory of Chiral Induced Spin Selectivity. Nano Lett. 2019, 19, 5253-5259.

(16) Onsager, L. Reciprocal Relations in Irreversible Processes. I. Phys. Rev. 1931, 37, 405-426.

(17) Onsager, L. Reciprocal Relations in Irreversible Processes. II. Phys. Rev. 1931, 38, 2265-2279.

(18) Büttiker, M. Symmetry of Electrical Conduction. IBM J. Res. Dev. 1988, 32, 317-334.

(19) Fransson, J. Vibrational Origin of Exchange Splitting and Chirality Induced Spin Selectivity. Phys. Rev. B 2020, 102, 235416.

(20) Fransson, J. Chirality-Induced Spin Selectivity: The Role of Electron Correlations. J. Phys. Chem. Lett. 2019, 10, 7126-7132.

(21) Sánchez, D.; Büttiker, M. Magnetic-Field Asymmetry of Nonlinear Mesoscopic Transport. Phys. Rev. Lett. 2004, 93, 106802.

(22) Büttiker, M.; Sánchez, D. Interaction-Induced Magnetic Field Asymmetry of Nonlinear Mesoscopic Electrical Transport. Int. J. Quantum Chem. 2005, 105, 906-913.

(23) Spivak, B.; Zyuzin, A. Signature of the Electron-Electron Interaction in the Magnetic-Field Dependence of Nonlinear IV Characteristics in Mesoscopic Systems. Phys. Rev. Lett. 2004, 93, 226801.

(24) Rikken, G. L. J. A.; Fölling, J.; Wyder, P. Electrical Magnetochiral Anisotropy. Phys. Rev. Lett. 2002, 87, 236602.

(25) Saito, K.; Utsumi, Y. Symmetry in Full Counting Statistics, Fluctuation Theorem, and Relations Among Nonlinear Transport Coefficients in the Presence of a Magnetic Field. Phys. Rev. B: Condens. Matter Mater. Phys. 2008, 78, 115429.

(26) Papaconstantopoulos, D. A. Handbook of the Band Structure of Elemental Solids, 2nd ed.; Springer New York Heidelberg Dordrecht London, 2015; pp 295-299.

(27) Choi, K. Theoretical Report On the Hopping Between Gold and Sulfur in CISS Experiments, MA Thesis, Delft University of Technology, 2020.

(28) Groth, C. W.; Wimmer, M.; Akhmerov, A. R.; Waintal, X. Kwant: A Software Package for Quantum Transport. New J. Phys. 2014, 16, 063065.

(29) Bedkihal, S.; Bandyopadhyay, M.; Segal, D. The Probe Technique far From Equilibrium: Magnetic Field Symmetries of Nonlinear Transport. Eur. J. Phys. B 2013, 86, 506.

(30) Ben Dor, O.; Yochelis, S.; Radko, A.; Vankayala, K.; Capua, E.; Capua, A.; Yang, S.-H.; Baczewski, L. T.; Parkin, S. S. P.; Naaman, R.; Paltiel, Y. Magnetization Switching in Ferromagnets by Adsorbed Chiral Molecules Without Current or External Magnetic Field. Nat. Commun. 2017, 8, 14567. 